\title{
Think tanks de derecha y discursos de género en Chile
}

\author{
Right-wing think tanks and gender discourses in Chile
}

Think tanks de direita e discursos de gênero no Chile

\section{Gina Paola Rodríguez*}

\section{RESUMEN}

Este trabajo reconstruye y estudia los repertorios discursivos en torno a la temática de mujer y género de tres think tanks de derecha chilenos: Libertad y Desarrollo, Fundación para el Progreso y Avanza Chile. A partir del análisis crítico de un corpus documental compuesto por informes, columnas de opinión e intervenciones en medios de comunicación de los expertos pertenecientes a estos tres centros, deseamos aportar en la comprensión de cuestiones como las siguientes: ¿Cómo entender la creciente incorporación de tópicos del feminismo dentro de la agenda de los think tanks de la derecha chilena? ¿Cuáles son los contornos conceptuales y los referentes del feminismo retomados por dichas organizaciones? ¿Cómo se insertan estos discursos en el objetivo mayor de librar una "guerra cultural" contra la izquierda y el progresismo?

\begin{abstract}
This work reconstructs and studies the discursive repertoires around the theme of women and gender of three Chilean rightwing think tanks: Libertad y Desarrollo, Fundación para el Progreso Avanza Chile. Based on the critical analysis of a documentary corpus composed of reports, opinion columns, and media interventions by experts belonging to these three centers, we wish to contribute to the understanding of questions such as the
\end{abstract}

Palabras clave: Chile, derechas, discursos, género, think tanks.

Keywords: Chile, right-wing, discourses, gender, think tanks.

\footnotetext{
* Argentina. Doctora en Ciencias Sociales de la Universidad de Buenos Aires. Centro de Investigaciones en Ciencias Jurídicas de la Universidad Nacional de La Pampa e Instituto de Estudios de América Latina y el Caribe, IEALC de la Universidad de Buenos Aires. Santa Rosa, La Pampa, Argentina. E-mail: gprodriguezm@unlpam.edu.ar
} 
following: How to understand the growing incorporation of topics of feminism within the agenda of the Chilean right-wing think tanks? What are the conceptual contours and references of feminism taken up by these organizations? How do these discourses fit into the larger objective of waging a "cultural war" against the left and progressivism?

\section{RESUMO}

Este trabalho reconstrói e estuda os repertórios discursivos em Palavras-chave: torno da temática de mulher e gênero de três think tanks chileChile, direitas, nos de direita: Libertad y Desarrollo, Fundación para el Progreso e Avanza Chile. Com base na análise crítica de um corpus docudiscursos, gênero, think tanks. mental composto por relatórios, colunas de opinião e intervenções em meios de comunicação dos especialistas pertencentes a esses três centros, desejamos contribuir para a compreensão de questões como as seguintes: Como entender a crescente incorporação de temas de feminismo na agenda dos think tanks da direita chilena? Quais são os contornos conceituais e as referências do feminismo adotadas por essas organizações? Como esses discursos se encaixam no objetivo maior de travar uma "guerra cultural" contra a esquerda e o progressismo? 


\section{Introducción}

Los think tanks o tanques de pensamiento (en adelante TT, por sus siglas en inglés) o centros de pensamiento han adquirido una importancia creciente en la sociedad chilena desde los años noventa. Una nueva generación de organizaciones vinculadas directa o indirectamente al mundo político, a sectores empresariales o a una combinación de ambos, hicieron presencia en el espacio público con el objetivo de proveer ideas y discursos para la toma de decisiones en el ámbito estatal.

El regreso a la democracia estuvo marcado por el desarrollo de una clase política experta, altamente calificada, a la que se encomendó la administración del Estado. Tecnócratas y technopols, ${ }^{1}$ jugaron un papel relevante en la configuración de la administración pública de los gobiernos de la Concertación (1990-2010) y en la oposición al primer mandato de Sebastián Piñera (2010-2014), ya fuera en la conformación de la agenda pública, el análisis y formulación de políticas, o bien, en el reclutamiento de cuadros burocráticos (González Bustamante, 2013; Silva, 2010). Pero no solo los gobiernos de centro-izquierda acudieron a la experticia de los TT, también las dos administraciones de Sebastián Piñera (2010-2014 y 2018-) han incluido en sus gabinetes a integrantes de fundaciones y centros de pensamiento liberal-conservadores y libertarios, cuya influencia ha ido en constante aumento, dada su cercanía con el sector representado por el piñerismo: los grandes grupos empresariales (Gárate, 2008; Olivares, González-Bustamante, Meneses y Rodríguez, 2015).

Buena parte de la producción académica referente a los TT en Chile se ocupa de los aspectos conceptuales de la categoría, sus tipologías, características y productos (Moreno, 2015; Pinilla, 2012). Cociña y Toro (2009) reconstruyen el ciclo de vida de los TT chilenos desde la dictadura y observan su relación con el sistema de partidos. Gárate (2008), en tanto, visualiza el espectro ideológico de estos centros en la década del 2000 clasificándolos, según sus intereses y tendencias, en conser-

1 Los tecnopols son definidos por Dominguez (1996) como un tipo de agentes que posee, al mismo tiempo, recursos técnicos obtenidos en centros académicos internacionales de excelencia, y recursos políticos inherentes a la ocupación de posiciones partidarias estratégicas previas a su nombramiento en cargos del Estado. Al poseer capital tanto intelectual como político, se trata de agentes dominantes en ambos campos. 
vadores, liberales socialcristianos y liberales progresistas ${ }^{2}$. Por su parte, Olivares et al. (2015) estudian las condiciones en las cuales la pertenencia a un centro de pensamiento se vuelve un factor determinante para acceder al gabinete ministerial durante los dos gobiernos de Bachelet y el primero de Piñera. Fisher y Plehwe (2013) toman distancia del caso chileno para ofrecer una mirada global de los TT neoliberales asociados a la Red Atlas en América Latina. Finalmente, Gartenlaub (2018) se refiere a los tanques de pensamiento en el marco de un estudio ampliado de los partidos de derecha en la posdictadura chilena.

De este breve estado de la cuestión colegimos que la producción dedicada especialmente a los think tanks de derecha (en adelante TTD) en Chile es relativamente escasa y la mirada acerca de sus discursos en torno al género prácticamente inexistente. Aprovechando esta vacancia, el presente trabajo se propone estudiar el repertorio de tres centros vinculados estrechamente con el piñerismo: Libertad y Desarrollo, Fundación para el Progreso y Avanza Chile. A partir del análisis crítico de un corpus documental compuesto por informes, columnas de opinión e intervenciones en medios de comunicación de los expertos de estos TTD, deseamos aportar conocimientos que nos permitan avanzar en la comprensión de cuestiones como las siguientes: ¿Cómo entender la creciente incorporación de tópicos del feminismo dentro de la agenda de los think tanks de la derecha chilena? ¿Cuáles son los temas y referentes conceptuales del feminismo retomados por dichas organizaciones? ¿Cómo se insertan en el objetivo mayor de librar una "guerra cultural" contra la izquierda y el progresismo?

En nuestra lectura, los TDD vienen dedicando parte de su batería argumental - tradicionalmente enfocada en temas de la experticia económica y el derecho- a la producción de discursos referente a algunos tópicos de la agenda feminista, en la idea de dotar a los sectores que representan de guiones persuasivos y polémicos con alta capacidad de impacto en la opinión pública. Este desplazamiento se enmarca en el objetivo mayor de librar una "guerra cultural" contra la izquierda y el progresismo y responder a la ola de demandas de cambio estructural exigida por los feminismos chilenos, entre otros movimientos sociales.

2 Gárate no incluye en su análisis a aquellas organizaciones ligadas a la izquierda extraparlamentaria. 
Entendemos que el lenguaje no es únicamente un medio para expresar y reflejar nuestras ideas, sino un factor que participa y tiene injerencia en la constitución de la realidad social. Esta concepción activa nos permite entender lo discursivo como un modo de acción. Los discursos son observables y constituyen una base empírica a partir de la cual conocer la realidad. Esto no implica que los discursos sean transparentes o que sean un calco de la realidad (reificación). Sin embargo, permiten acceder a los modos en que los distintos actores sociales dan forma a sus sistemas de ideas y representaciones.

Estructuramos este artículo en dos apartados. En el primero, elaboramos una reconstrucción de las trayectorias y perfiles ideológicos de los TTD seleccionados y sus gestores-expertos. A continuación, relevamos y analizamos sus principales líneas discursivas en relación con tres agrupamientos o nodos críticos de las luchas por la justicia de los feminismos: la redistribución económica, la representación política y el reconocimiento simbólico de las mujeres y las disidencias sexogenéricas. A lo largo del trabajo trataremos de establecer posibles conexiones entre las ideas y recomendaciones de los TTD y la "Agenda mujer" de la segunda administración de Piñera.

\section{Trayectorias y perfiles ideológicos de los TTD}

Sebastián Piñera encabezó el primer gobierno de derecha democráticamente elegido en Chile desde 1958. En tiempos de campaña, se presentó como una posible renovación política y una inflexión en el proceso de transición administrando el Estado según el modelo de gestión del sector privado (Schuster y Morales, 2015). Al poco tiempo de su posesión, este cometido fue cuestionado por movimientos sociales masivos que irrumpieron en la arena pública para recusar las estructuras de la sociedad chilena posdictadura (Mira, 2011; Silva, 2010).

De cara a la reelección, Piñera se vio en la necesidad de atraer actores de la sociedad civil para reforzar su sistema de alianzas y relanzar su proyecto programático. Si bien se abocó a la búsqueda de capital cultural y puestos para la segunda línea de gobierno con un énfasis técnico, el criterio de selección de colaboradores siguió más asociado con la participación activa en la campaña presidencial que a la vinculación partidaria. Como ocurrió con el primer gabinete, los ministros elegidos para la segunda administración de Piñera replica- 
ban el perfil profesional, económico y la trayectoria del propio presidente (Varas, 2013).

Fuera por la vía de la experticia, la militancia o la identidad de clase, los TT más favorecidos con cargos en el segundo gobierno de Piñera fueron fundamentalmente tres: Libertad y Desarrollo (LyD), Fundación para el Progreso (FPP) y Avanza Chile. A continuación reconstruimos su trayectoria y perfil ideológico.

\section{Libertad y Desarrollo}

Se trata de un centro de estudios e investigación privado creado en 1990 por tres exministros de Pinochet - Hernán Büchi³ ${ }^{3}$ Carlos F. Cáceres $^{4}$ y Luis Larraín Arroyo ${ }^{5}$ - y por el actual jefe de asesores de la Presidencia de Sebastián Piñera, Cristián Larroulet ${ }^{6}$. Si bien este tanque de pensamiento se declara como una institución independiente de todo grupo político, religioso, empresarial y gubernamental, se advierte la presencia de varios de sus expertos en distintas campañas presidenciales y cargos gubernamentales?

3 Büchi es ingeniero civil de la Universidad de Chile y realizó sus estudios de posgrado en la Universidad de Columbia, Nueva York. Se ha desempeñado como subsecretario de Economía (1979-1980), subsecretario de Salud (1980-1983), ministro de Planificación (1983-1984), superintendente de Bancos e Instituciones Financieras (1984-1985) y ministro de Finanzas (1985-1989).

4 Cáceres es ingeniero comercial de la Universidad Católica de Valparaíso y MBA de Cornell University, con estudios de posgrado en Harvard Business School. Fue miembro del Consejo de Estado (1976), presidente del Banco Central (1982-1983), ministro de Hacienda (1983-1984) y ministro del Interior (1988-1990). Es miembro de la Sociedad Mont Pèlerin, además de director de empresas y profesor universitario.

5 Larraín es ingeniero comercial y licenciado en Economía de la Universidad Católica de Chile. Fue investigador de la publicación Economía y Sociedad (1978-1979), dirigida por José Piñera, hermano del actual presidente y conocido por su rol en la generación e implementación de reformas neoliberales durante la dictadura de Pinochet. Fue asesor del ministro del Trabajo y Previsión Social y miembro del Comité de Estudios de la Reforma Previsional, superintendente de Seguridad Social y ministro director de la Oficina de Planificación Nacional (1979-1980), entre otro cargos. Se desempeñó además como director de las empresas Chilectra, CTC, Colbún y Metro.

6 Larroulet es ingeniero comercial de la Pontificia Universidad Católica y Master of Arts en economía por la Universidad de Chicago. Fue macrocoordinador del grupo Tantauco que elaboró el programa de gobierno de la primera candidatura presidencial de Sebastián Piñera. En 2010, fue nombrado ministro secretario general de la Presidencia. En 2018, asumió como jefe de asesores de la Presidencia de la República durante la segunda administración de Sebastián Piñera

7 Además del fundador de LyD Cristián Larroulet, este sería el caso de varios consejeros e investigadores que ocupan u ocuparon cargos ministeriales en distintos momentos de las dos administraciones del presidente Piñera: Ena Von Baer, Marcela Cubillos, 
Cuenta con Consejo Asesor de Políticas Públicas compuesto por 12 expertos (nueve varones y tres mujeres); un equipo de 15 investigadores (siete varones y ocho mujeres) repartidos en siete programas: Económico, Social, Sociedad y Política, Legislativo, Medio Ambiente, y Justicia; y un área de comunicaciones (cuatro mujeres). Según áreas disciplinares, el equipo está formado por profesionales de las ciencias económicas (36,8\%), el derecho $(36,8 \%)$ y la comunicación social $(26,3 \%)$. Además, trabajan trece personas en el área de administración (10 mujeres y tres varones).

En cuanto al perfil ideológico, LyD puede calificarse como un TT liberal-conservador. Así lo expresa su objetivo de

colaborar para que las políticas públicas en Chile se orienten a defender la libertad individual, el libre funcionamiento de los mercados, el derecho de propiedad y el progreso e igualdad de oportunidades de sus habitantes a través del desarrollo económico. (LyD, 2020b).

Junto al laissez faire económico, LyD suscribe valores conservadores tradicionales como el rechazo del concepto de justicia social - ante la creencia de que la desigualdad social es natural y hasta fructífera- y la defensa de la familia tradicional como unidad básica de la sociedad y foco del desarrollo (LyD, 2018).

Las orientaciones impartidas a los profesionales de LyD para realizar su labor, exponen con mayor detalle los valores ético-políticos del centro:

Un estado moderno requiere de un gobierno que genera las reglas del juego para que las personas desarrollen sus potenciales en un ambiente propicio para la colaboración, el emprendimiento y el respeto por los derechos de los demás. La acción de dicho gobierno debiera enmarcarse en el principio de subsidiariedad, abordando este, solo aquellas tareas que los individuos, por sí solos o actuando asociativamente en las organizaciones que decidan darse, no sean capaces de emprender. Así, la regulación deberá procurar incentivar más que obligar, disuadir más que prohibir. (LyD, 2020b). 
La defensa de un Estado que interviene solo excepcionalmente en la sociedad dejando librado el juego a las fuerzas del mercado y el sector privado es el primero de los principios orientadores del LyD. Todas las regulaciones, insisten, deberán seguir un criterio económico: el análisis de costo-beneficio.

La segunda orientación para sus integrantes es el análisis técnico de las políticas públicas. Los temas por investigar no surgen por iniciativa de los expertos, ni tampoco sus metodologías. En su lugar, realizarán análisis técnicos de las áreas que el director ejecutivo y los jefes les asignen para su estudio. Estos criterios marcan una diferencia sustancial respecto de los TT universitarios, donde la libertad de los investigadores es relativamente mayor. Se trata, siguiendo la clasificación de Weaver (1989) de un instituto de investigación bajo contrato que, si bien sigue cierto rigor metodológico como los centros universitarios, orienta su repertorio de problemas según las necesidades de sus fuentes de financiamiento, las cuales provienen de contratos con empresas privadas y aportes de fundaciones internacionales ideológicamente afines.

La tercera orientación para los expertos del LyD impele al trabajo en redes nacionales e internacionales de apoyo a las ideas del liberalismo económico con fines de difusión. Esto incluye el intercambio con medios de comunicación, partidos políticos, Gobierno, Congreso, instituciones académicas y centros de estudios o pensamiento, instituciones religiosas o del ámbito de la moral, empresas, gremios, sindicatos, y otros grupos de interés particular (LyD, 2020b).

El énfasis divulgativo se traduce en la publicación de un abultado número de libros (355, según el catálogo en línea) bajo su propio sello editorial y la aparición frecuente de sus miembros en medios de comunicación a través de columnas de opinión, entrevistas e interacción en redes sociales. El trabajo en red se refleja en su articulación con otros TT nacionales ${ }^{8}$ e internacionales ${ }^{9}$ y su participación en conglomerados neoliberales como la Red Liberal de América Latina (RELIAL) y la Red Atlas.

8 Entre ellos: Fundación para el Progreso, Fundación Avanza Chile, Instituto Res Pública, Fundación Jaime Guzmán, Instituto Libertad y Horizontal Chile.

9 Tales como Cedice Venezuela, Centro Hispanoamericano para la Investigación Económica, Fundación Libertad, The Cato Institute, The Heritage Foundation. 
Resumiendo hasta aquí, podemos decir que LyD surge en el segundo ciclo de los TT en Chile, y es el de mayor trayectoria de los tres que analizaremos en este trabajo con un perfil marcadamente técnicopolítico. A nivel ideológico es un instituto conservador en lo social (en línea de continuidad con la derecha pinochetista), defensor del liberalismo económico desregulado y de los intereses de las grandes empresas.

\section{Fundación para el Progreso (FPP)}

Esta fundación se autodefine como un centro de estudios liberal, independiente y sin fines de lucro, formado por profesionales jóvenes, líderes de opinión, académicos e intelectuales públicos en Chile. Fue creada en 2012 por el empresario Nicolás Ibáñez, con la misión de

impulsar un cambio cultural que promueva las bases de una sociedad más próspera, libre, digna, inclusiva y en paz; a través de la difusión del ideario liberal en espacios de influencia y la formación de jóvenes líderes que guíen a Chile y América Latina por el camino del progreso. (FPP, 2020).

Además del Grupo Drake, propiedad de Nicolás Ibáñez, la FPP recibe financiamiento del holding Ultramar dirigido por Dag Von Appen, nieto del líder de las operaciones nazis en América Latina Julio Alberto Von Appen (Jerez, 2018).

A nivel ideológico, la FPP suscribe valores propios de la derecha libertariana:

Creemos que el progreso es el descubrimiento de aquello que aún no conocemos y que ese descubrimiento solo puede darse en espacios de profunda libertad.

El potencial humano florece únicamente cuando a las personas les resulta posible desplegar libremente su singularidad, en un juego espontáneo de colaboración voluntaria y pacífica.

En FPP vivimos cada día, cada minuto, los principios y valores de una sociedad libre. Creemos en el valor de la persona humana, la libertad y responsabilidad individual, el respeto, tolerancia y diversidad entre las personas. Luchamos por una sociedad civil empoderada, una economía libre y la cooperación entre las personas. 
Buscamos la paz, el escepticismo frente al poder, un estado de derecho, interacciones voluntarias y acuerdos libres y abogamos por un estado limitado y gobiernos responsables. (FPP, 2020, negrillas en el original).

Como hemos visto en otro trabajo,

para los libertarianos una sociedad justa supone la capacidad para asegurar las condiciones que permitan proteger los derechos y libertades civiles de sus miembros, y particularmente, el derecho a la propiedad privada. Por ello, el papel conferido al individuo es esencial. (Rodríguez, 2013, p. 80).

Cualquier tipo de relación social o política está precedida por los intereses individuales, configurándose el dualismo entre moral individual y moral social, entre vida privada y vida pública, o entre moral y política. Esta base ontológica explica "la defensa de un individualismo radical, donde la diferencia como desigualdad constituye la razón central para abogar por un Estado que apenas se ocupe de garantizar y proporcionar la seguridad de los asociados" (Rodríguez, 2013, p. 81).

En cuanto a su estructura orgánica, la FPP se compone por un consejo directivo de siete miembros (seis varones y una mujer); tres consejos regionales (Valparaíso: dos miembros, Concepción: cinco miembros y Valdivia: cinco miembros); tres senior fellows ${ }^{10}$ (todos varones); tres miembros del equipo de gerencia (todos varones) y un total de 30 personas (18 varones y 12 mujeres) distribuidas en tareas de formación y extensión, investigación y publicaciones, soporte, comunicaciones, arte e imagen, relaciones internacionales, gestión de personas, producción general y administración.

A diferencia de LyD, cuyos consejeros e investigadores son technopols experimentados, los integrantes de la FPP son en su mayoría jóvenes profesionales y universitarios que actúan en Santiago, la Quinta Región (Valparaíso, Concepción) y Valdivia y concentran su función en la "formación de una nueva generación liberal" y el fomento de este

10 Denominación dada a los miembros que ocupan las posiciones más altas dentro del TT debido a su mayor trayectoria académica y/o experticia. El témino original es usado en el mundo universitario de habla inglesa para referir a los investigadores o miembros de corporaciones académicas con las más altas credenciales. 
ideario a través de la "participación en la discusión pública y los espacios de influencia cultural” (FPP, 2020).

Los senior fellows son Axel Kaiser, Gonzalo Sanhueza y Roberto Ampuero. Kaiser ${ }^{11}$ es la cara más visible de la FPP, no solo por su condición de director ejecutivo, sino por el estilo polémico de sus libros ${ }^{12}$ e intervenciones públicas donde defiende ideas como la justificación de la desigualdad natural, la libertad negativa y el Estado mínimo, en contra de los sectores liberales que defienden el principio de subsidiaridad $^{13}$.

La trayectoria política de Roberto Ampuero ${ }^{14}$ es llamativa, pues pasó de militar en las Juventudes Comunistas y exiliarse en Alemania Oriental durante la dictadura de Pinochet a apoyar abiertamente las candidaturas de Eduardo Frei y Sebastián Piñera en los años 2000. En los gobiernos de este último ocupó los cargos de ministro de Cultura (2013-2014), ministro de Relaciones Exteriores (2018-2019) y embajador de Chile en España (2019-actualidad).

Además de Ampuero, otro integrante de la FPP que llegó al gobierno de Piñera fue Gerardo Varela, un abogado que se desempeñó como ministro de Educación durante cinco meses (marzo-agosto de 2018). Su paso por el Gabinete fue, además de corto, muy criticado por cuenta de sus declaraciones públicas. Dichos como que la Educación es un derecho y también un bien económico o que los daños en la infraestructura de los colegios debían ser financiados a través de bingos, le valieron finalmente la remoción del cargo (Reyes, 2018).

11 Abogado y doctor en Filosofía por la Universidad de Heidelberg (Alemania), profesor de la cátedra Friedrich von Hayek de la Universidad Adolfo Ibáñez (propiedad del presidente de la FPP) y columnista habitual de El Mercurio, El Líbero y el Diario Financiero.

12 Entre los que contamos: El Chile que viene (2007), La fatal ignorancia (2009), La miseria del intervencionismo (2012), La tiranía de la igualdad (2015), El engaño populista (2016) y El Papa y el capitalismo (2018).

13 Como observan Alenda, Foulon, y Suárez-Cao (2019) en el caso de Chile, el principio de subsidiariedad sin vincularse unívocamente al liberalismo o al conservadurismo, contribuye a forjar cierta sensibilidad respecto al lugar que el Estado debe ocupar en la economía y se articula con la moral cristiana, mediante una interpretación tradicionalista de la doctrina neoliberal, materializada en la noción de "subsidiarismo católico".

14 Escritor, licenciado en Literatura Hispánica por la Universidad de La Habana, Cuba; egresado de la Escuela Superior Política Wilhelm Pieck, Berlín, Alemania Oriental; Master of Arts en Literatura Latinoamericana y Doctor en Filosofía en cultura hispánica por la Universidad de Iowa, EE.UU. 
La presencia de Ampuero en la cartera de Cultura y de Varela en Educación se enmarca en la estrategia mayor de la FPP: librar una "guerra de ideas" contra el progresismo. Axel Kaiser, se ha referido a este imperativo en los siguientes términos:

La de las ideas, sugirió Hayek, es la madre de todas las batallas ... Hayek y Fisher entendieron que si no cuenta con intelectuales y defensores que lo legitimen y prestigien, el sistema de libre empresa no puede subsistir. Y es que, como ha afirmado el Nobel de Economía Douglas North, no basta que la gente se beneficie del sistema: además debe creer en él. Quien pretenda defender el capitalismo no puede olvidarse de las ideas ni de la lucha cultural. De lo contrario, como advierte North, el sistema de libre empresa se encuentra a merced de las ideologías, que son "materias de fe antes que de razón y subsisten pese a las abrumadoras pruebas en contrario." (Kaiser, 2011).

Mientras la noción gramsciana de batalla cultural denota una conceptualización crítica del diálogo conflictivo en el que cada bloque de poder intenta apropiarse de la palabra del otro, y cuyo triunfo hegemónico es precisamente el ocultamiento del conflicto; la "guerra de ideas" invocada por Kaiser, correspondería solo al momento hegemónico, prescribiendo la supresión de las ideas del otro en nombre de un acto científico o libre de ideología. La forma de esta supresión es la estigmatización de las ideas "progresistas" o de izquierda como ideologías (en el sentido de falsa conciencia) o materias de fe. Ante estas, la doxa neoliberal se presenta a sí misma como neutral y avalorativa, y sus argumentos como frutos de la razón asépticos y no ideológicos. Si entendemos con Althusser (1988) que no hay un afuera de la ideología, la apelación al carácter incontaminado y científico del liberalismo resulta ser el más refinado de sus artificios ideológicos.

Más allá de la reflexión filosófica nos interesa comprender los modos concretos de operación de la guerra de ideas a través de los think tanks. A la luz del relato elaborado por Kaiser, este combate se libra en Chile en respuesta a la avanzada del progresismo que ha puesto en peligro la credibilidad en el sistema de la libre empresa:

En Chile, por ejemplo, la ideología ha avanzado de tal manera, que está poniendo en jaque la credibilidad del sistema de libre em- 
presa. La razón es que no se libró en su momento la batalla de las ideas. Una visión reduccionista del ser humano llevó a asumir que mientras hubiera consumo no había de qué preocuparse. Como resultado, hoy, los que sí hicieron el trabajo de penetración cultural han logrado minar peligrosamente la credibilidad del modelo. Se ha llegado al punto de que la palabra empresario es prácticamente sinónimo de sinvergüenza. Y lo más preocupante es que quienes pueden dar la lucha por las ideas no reaccionan. Parecen no ver que lo que necesitamos para contrarrestar esta nefasta evolución son líderes de la talla de Sir Anthony Fisher. (Kaiser, 2011).

Ante este estado de situación, el papel de la FPP y sus miembros es claro: descentralizar el debate de las ideas y la reflexión en torno a la sociedad libre a través de la puesta en marcha de un plan de expansión regional mediante el trabajo colaborativo con organizaciones a lo largo del país y el acceso a contenidos gracias a cursos online, recursos digitales y actividades (FPP, 2020).

Sobre la base de lo anterior, y siguiendo la clasificación de TT sugerida por Weaver (1989), encontramos que la FPP puede tipificarse como un centro militante. No solo por su aparición reciente en el ámbito público (en contraste con TT consolidados como LyD), sino fundamentalmente por integrar un activo componente ideológico y partidista con un poderoso aparato publicitario orientado a impactar en el debate público. Antes que a la producción de datos y estudios originales, la FPP se dedica a impulsar temas en la agenda pública usando los resultados de otras investigaciones en infografías y documentos breves y de fácil lectura con el objetivo de influir en los decisores políticos. De este modo, más que dar recomendaciones de políticas públicas como el LyD, la FPP le apuesta a la guerra de ideas en medios de comunicación de su competencia en el mercado de bienes simbólicos.

Hasta este punto, podemos decir que aunque LyD es un TT claramente neoliberal, aparece como moderado frente a la libertariana FPP. Además de manejar un presupuesto importante, la FPP cuenta con una red internacional de contactos con las fundaciones y centros neoliberales más influyentes del mundo, como el Ayn Rand Institute y la Atlas Society, que están aún más a la derecha del Partido Republicano de los EE.UU., proponiendo la total disolución del Estado. 


\section{Avanza Chile}

Esta fundación debe su origen a Sebastián Piñera quien, tras terminar su primera presidencia, vio la necesidad de crear un frente político de oposición para los años posteriores. Como expertos gestores y miembros del directorio de Avanza Chile se destacan cuadros de la primera línea del piñerismo como Andrés Chadwick ${ }^{15}$, Cecilia Pérez ${ }^{16}$, Gonzalo Blumen ${ }^{17}$, Alfredo Moreno ${ }^{18}$ e Isabel Plá ${ }^{19}$.

Según describe en su página web, Avanza Chile se trazó como misión "contribuir al debate de las grandes ideas y políticas públicas para expandir las libertades y oportunidades de todos los chilenos y avanzar hacia una sociedad más democrática, justa, pluralista y fraternal" (Avanza Chile, 2020). A nivel ideológico podemos clasificarlo como un TT liberal de centro-derecha, que defiende la idea de subsidiariedad en la acción estatal, en consonancia con el objetivo de renovación programática y adaptación estratégica a partir del cual el piñerismo ha buscado ser más competitivo electoralmente. Así, puede leerse entre sus objetivos:

Estudiar, difundir, promover y defender las ideas, valores y principios de la libertad, la democracia, los derechos humanos, la justicia, la igualdad de oportunidades, el pluralismo y el progreso, así como también las ideas, valores y principios de la vida, la dignidad

15 Abogado participante de la Junta Militar de Augusto Pinochet, y militante del partido Unión Demócrata Independiente (UDI), pasó de ser presidente del directorio de Avanza Chile a ministro del Interior y Seguridad Pública, entre marzo de 2018 y octubre de 2019. Durante su gestión tuvo lugar el asesinato del comunero mapuche Camilo Catrillanca y la represión de las protestas sociales de octubre de 2019. En diciembre del mismo año, el Senado chileno lo responsabilizó políticamente por las violaciones a los derechos humanos cometidas por Carabineros y Ejército y lo inhabilitó para ejercer cargos públicos hasta 2024 .

16 Vicepresidenta de Avanza Chile, es una abogada y política militante de Renovación Nacional (RN), regresó al gabinete en marzo de 2018 como ministra de la Secretaría General de Gobierno y se mantuvo en el cargo hasta el 28 de octubre de 2019. Tras las masivas protestas de ese mes fue nombrada como ministra del Deporte.

17 Militante de Evolución Política (Evópoli) y exinvestigador de LyD, pasó de director ejecutivo de Avanza Chile a secretario general de la Presidencia (hasta octubre de 2019) y después ministro del Interior y Seguridad Pública en reemplazo de Andrés Chadwick (hasta el 28 de julio de 2020).

18 Economista, ministro de Relaciones Exteriores del primer gobierno de Piñera, se desempeñó como ministro de Desarrollo Social (marzo 2018-junio 2019) y como ministro de Obras Públicas (junio 2019).

19 Militante de la UDI. Isabel Plá, ocupó el Ministerio de la Mujer y Equidad de Género (marzo 2018- marzo 2020). 
de las personas, la familia, la paz, la solidaridad y el desarrollo sustentable. (Avanza Chile, 2020).

Como explica Rovira Kaltwasser (2019), esta adaptación responde a las transformaciones de la sociedad chilena — cada vez más liberal en términos morales y demandante de un Estado con un rol activo en la generación de bienestar- que han obligado a la derecha a repensar su agenda programática. Según el autor, buena parte de la competitividad electoral que ha ganado la derecha chilena se debe a su contemporización con las demandas de la ciudadanía y a "su gradual distanciamiento de una agenda inicialmente marcada por una defensa irrestricta del libre mercado" (Rovira Kaltwasser, 2019, p. 31), aunque coincidimos en el diagnóstico de la "(sobre)adaptación estratégica" con fines electorales, creemos que el mentado distanciamiento de la agenda del libre mercado no es tal. Conquistas sociales como la gratuidad educativa o la ley de identidad de género, logradas durante el piñerismo son el resultado de una movilización ciudadana que logró eco en el Congreso y no el producto de la iniciativa presidencial o de los sectores políticos que la circundan. La resistencia del piñerismo a avanzar más allá de lo cosmético se hace evidente, además, en su "Agenda Mujer" que mantiene inalterados los arreglos institucionales que perpetúan la desigualdad y la violencia hacia las mujeres y omite flagrantemente cualquier política en relación con las disidencias sexo-genéricas.

Allende estos límites, es cierto que el TT presidencial aparece como adalid de un liberalismo moderado y dialoguista en comparación con el conservadurismo social de LyD y el anarcocapitalismo de FPP. Las acciones de Avanza Chile que buscan concretar el objetivo de ampliar su base electoral incluyen el fomento de proyectos de creación, promoción, investigación y difusión de la cultura, las artes, las ciencias y la protección del medio ambiente; el debate de ideas y proyectos en el campo internacional y el sostenimiento de relaciones públicas y colaboraciones con organizaciones equivalentes a nivel nacional e internacional (Avanza Chile, 2020). En esta última perspectiva, se destacan reuniones con personajes de la derecha política internacional como la líder opositora cubana Yoani Sánchez y las esposas de Leopoldo López y Antonio Ledezma, presos políticos de la oposición venezolana. 
Retomando el sistema clasificatorio de Weaver (1989), podemos decir que Avanza Chile puede ser considerado como un TT organizado en torno a un partido político. Aunque se autodefine como intelectualmente autónoma, el trabajo de la fundación es directamente útil al piñerismo, proveyendo discursos de oposición en tiempos del gobierno de Bachelet y cuadros burocráticos una vez en el poder. La relación del organismo es casi mimética con las elites partidarias, los ministerios y los representantes elegidos. Esto puede explicar por qué el grueso de su actividad se detiene a finales 2017, poco antes de que Sebastián Piñera arribara por segunda vez a La Moneda. Desde entonces, sus principales miembros pasaron a ofrecer su experticia directamente en el Estado.

Si comparamos la participación de los tres centros de estudio en la conformación del gobierno de Piñera, observamos que Avanza Chile es el que ha logrado mayor presencia. Sus miembros han estado en la primera línea política y algunos incluso han persistido después de los cambios de gabinete. El traspaso de los cuadros técnicos de este TT al Gobierno, confirman que Avanza Chile fungió como un espacio de reclutamiento y concentración de la masa burocrática requerida por la coalición de Chile Vamos.

\section{Los Think Tanks de derecha y la lucha feminista}

Durante el primer gobierno de Piñera las políticas públicas privilegiaron a la familia tradicional como sujeto de derechos y no a las propias mujeres, quienes solo fueron atendidas a través de programas focalizados dirigidos a mejorar la empleabilidad de las llamadas poblaciones vulnerables. Bajo esta comprensión, los problemas de las mujeres eran vistos como producto de la pobreza y no de las relaciones de género. En concordancia, el reajuste de la institucionalidad estatal creada por la Concertación para proponer y coordinar las políticas de equidad de género, se orientó a la despolitización del Servicio Nacional de la Mujer y a la invisibilización de la dominación patriarcal (Álvarez y Navarrete, 2019; Torres, 2011).

Cuando Piñera asumió su segundo mandato, los movimientos de mujeres y disidencias sexo-genéricas ya habían advertido la persistencia de hondas limitaciones institucionales para la garantía efectiva de sus derechos, no obstante los avances logrados durante la adminis- 
tración Bachelet (2014-2018) ${ }^{20}$. Universidades, institutos y colegios en tomas y paros exigieron de forma urgente el fin de los abusos y acosos sexuales, sanciones para los victimarios, el fin de la educación sexista y una agenda feminista nacional.

Desde noviembre de 2018, miles de mujeres y organizaciones a lo largo del territorio perfilaron las discusiones que meses después dieron lugar a la Primera Huelga General Feminista que movilizó a más de 300 mil personas en Santiago y 800 mil a nivel nacional. Las demandas y acuerdos de la huelga se sintetizaron en diez puntos: fin de la violencia machista; derecho a la ciudad y la vivienda digna; memoria feminista y derechos humanos; trabajo y seguridad social; lucha por el aborto y derechos sexuales y reproductivos; racismo y migración; arte, cultura y comunicaciones; defensa del territorio, soberanía alimentaria, vida y territorios indígenas; disidencias sexuales y educación no sexista (Encuentro plurinacional, 2019).

En lectura de esta situación, el Ejecutivo lanzó en abril de 2019 la "Agenda Mujer", una batería de 22 reformas y leyes con las que intentó dar respuesta a la ola feminista que avanzaba ${ }^{21}$. La Segunda Huelga General Feminista, convocada para el 8 de marzo de 2020, no solo denunció el carácter incompleto e incumplido de la "Agenda Mujer", sino que amplió el decálogo de propuestas del feminismo plurinacional chileno a 16 ejes de demanda pensados en clave constitucional. Con estas vindicaciones, las feministas chilenas se pusieron en sintonía con la ola de protestas sociales iniciada en octubre de 2019, para remarcar el agotamiento del modelo político de la Concertación —que gestionó, sin fisurar, el legado de la dictadura - y el descontento social con aspectos estructurales de la sociedad y la institucionalidad.

20 Entre los que cabe mencionar la creación del Ministerio de la Mujer y la Equidad de Género y la Ley de Cuotas para estimular la inclusión de mujeres en las candidaturas a los cargos de representación popular.

21 Se trata de 22 medidas presentadas por Piñera el 23 de mayo de 2018, entre las que se incluyen: Reforma constitucional para "promover y garantizar la plena igualdad de derechos, deberes y dignidad entre el hombre y la mujer y para evitar toda forma de abuso o discriminación arbitraria contra las mujeres". Proyectos de ley: de derecho a sala cuna universal, de fuero maternal para mujeres que integren las Fuerzas Armadas, de sanción para la violencia en el pololeo, de lactancia libre, para terminar con las diferencias de precios en los planes de salud, de teletrabajo, y tipificación penal del femicidio. A la fecha de cierre de este artículo solo se ha avanzado en ocho de los 22 compromisos (Román, 2020). 
Los llamados a un cambio social e institucional profundo interpelaron al núcleo de intereses, representaciones y valores de la derecha chilena, obligando a los TT afines a articular nuevas estrategias discursivas para la preservación del statu quo. Si hasta hace un lustro, la agenda de los TTD priorizaba temas como la economía, la educación, la lucha contra la corrupción y la inseguridad; ahora las intervenciones de sus miembros empezaron a ocuparse de tópicos como la paridad de género, los derechos de las parejas del mismo sexo, la salud sexual y (no) reproductiva y la violencia contra las mujeres, entre otros. En nuestra lectura, este desplazamiento en el repertorio tiene un carácter estratégico en el marco de la batalla de ideas abierta por las luchas por la inclusión y la diversidad, y como parte de la necesaria adaptación de la derecha partidaria a los tiempos posideológicos, "que exigen la toma de posiciones respecto de nuevas cuestiones coyunturales y relativas a valores" (Alenda et al., 2019)

A continuación, analizamos el contenido de una serie de informes institucionales, columnas de opinión y declaraciones en medios de comunicación producidos por expertos de LyD, FPP y Avanza Chile entre 2016 y 2020, con el fin de conocer algunos de los enunciados a partir de los cuales las derechas chilenas han intentado responder a la ola de movilización social liderada por el feminismo plurinacional.

Un esquema útil para sintetizar las demandas del movimiento heterogéneo y plural de los feminismos en Chile es el aportado por Fraser (2008) y su teoría de la justicia en tres dimensiones: la redistribución en la esfera económica, el reconocimiento en el ámbito sociocultural y la representación en lo político.

Con distintos énfasis, los TT analizados en nuestra investigación invocan la doxa liberal de la igualdad de oportunidades entre hombres y mujeres, al tiempo que buscan neutralizar las demandas de redistribución, reconocimiento y representación exigidas por los feminismos locales. Para ello, acuden a argumentos que van desde su inconveniencia económica (basados en un cálculo costo-beneficio), su carácter contrario a la "evidencia científica" o su abierta negación. En la base de estos tres enunciados se halla una premisa mayor: la conformidad con el orden de género existente. 


\section{Igualdad sin redistribución}

Sabemos que, en principio, la derecha ultraliberal es renuente a cualquier política redistributiva por considerarla una intervención inaceptable del Estado sobre los derechos de los individuos. Los TTD que analizamos coinciden con esta mirada incluso cuando en sus diagnósticos describen que la situación de las mujeres chilenas es desventajosa.

En un informe de 2016, LyD quiso demostrar que la extensión de la cobertura del Bono al Trabajo de la Mujer entregado por el Ministerio de Desarrollo Social, no aumentaba la tasa de ocupación y mostraba un efecto no significativo para las mujeres pertenecientes al tramo entre el 35\% y $40 \%$ más vulnerable. La recomendación de LyD fue restringir aún más este subsidio de desempleo, enfocándolo solo en los grupos que más lo necesiten siempre y cuando esto redunde en la generación de empleo (LyD, 2016). Vale decir que el informe es estrictamente cuantitativo y no hace ninguna alusión al acceso desigual de las mujeres al mercado de trabajo, ni a las causas estructurales de esta desigualdad.

En otro informe del año 2019, el mismo TT ahonda un poco más en estas cuestiones y elabora un diagnóstico de la situación de las mujeres en Chile que se resume en los siguientes términos:

El estudio parte efectuando un análisis sobre el tema mujer y desarrollo y cómo fue evolucionando en el tiempo y en qué forma fue beneficiando a la mujer que pudo ir cambiando su rol tradicional en el hogar y la familia para acceder al mundo laboral. Se reseña cómo los países que más se desarrollaron y basaron su crecimiento en políticas sustentadas mayoritariamente en economías de mercado, fueron favoreciendo a la mujer para alcanzar un mayor desarrollo laboral y personal. Luego se efectúa un análisis comparativo respecto de la mujer en Chile y otros países de similar o mayor desarrollo. Se constata que en participación laboral y generación de ingresos hemos avanzado bastante y en otros indicadores de salud y educación, estamos más avanzados que los demás países latinoamericanos. No obstante, aún nos falta un tanto para llegar al nivel de países de más alto desarrollo humano del mundo. Las mayores brechas se dan en los ámbitos de participación política y toma de decisiones. (Camhi, 2019, p. 5). 
Tres cuestiones nos llaman la atención en este estudio. En primer lugar, la construcción de una correlación directa entre el ingreso al mundo laboral y el cambio en el rol tradicional de la mujer, una lectura que pierde de vista que la mujer trabajadora promedio en Chile es, al mismo tiempo madre y esposa, con lo cual lejos de abandonar su rol tradicional, es impelida a cumplir una doble jornada de trabajo. En segundo lugar, la tesis según la cual los países que basaron su crecimiento en políticas de libre mercado favorecieron más a las mujeres: un argumento que, además de incomprobable a la luz de los datos arrojados por el informe, es falaz en su construcción lógica (petición de principio).

Finalmente, la afirmación de una mejora progresiva y ascendente de la situación de las mujeres en Chile a partir de cuatro grupos de indicadores (participación laboral, generación de ingresos, mortalidadfecundidad y nivel educativo), la cual pierde de vista males endémicos padecidos por las mujeres chilenas como la violencia física y simbólica y la discriminación en todas sus formas. Sorprende que, en vías de argumentar este supuesto mejoramiento, el informe de LyD destaque el uso de electrodomésticos como medio para liberar y empoderar a las mujeres, sin problematizar la responsabilidad exclusiva de las mujeres en las tareas de cuidado, como se expresa en la siguiente cita:

Otro estudio, en la misma línea, evidencia cómo los avances tecnológicos del siglo pasado, como nunca antes, permitieron que la mujer tradicionalmente a cargo de los quehaceres del hogar y del cuidado de los hijos, fuera liberando tiempo personal, en pro de una mayor disponibilidad de tiempo para el trabajo y otras actividades de su preferencia, aumentando así sus opciones. El mejoramiento de la calidad de vida fue permitiendo un mayor empoderamiento e independencia, que a su vez derivó en cambios culturales que aminoraron el machismo y otras formas de prejuicios contra la mujer. (Camhi, 2019, p. 7).

El encadenamiento de ideas de causalidad improbada visto en el párrafo anterior, se completa con una serie de datos acerca de las percepciones de los chilenos respecto del rol de la mujer en la familia y la sociedad, obtenidos de una encuesta aplicada por la Pontificia Universidad Católica. En esta se preguntó a los encuestados si estaban muy de acuerdo, de acuerdo, o en desacuerdo con las siguientes afir- 
maciones: "La familia se descuida si la mujer trabaja tiempo a completo"; "Una madre que trabaja tiene una relación igual de cercana con los hijos que si no trabaja" y "Ser dueña de casa es tan satisfactorio como tener un trabajo" (PUC, Encuesta Bicentenario 2018, citado por Camhi, 2019, p. 24).

El sistema de representaciones de LyD se refleja en sus modos de indagar por el papel de la mujer. Las tres opciones de respuesta se ajustan a una concepción tradicional del orden de género, donde la mujer sujeta de derechos es aquella que decide maternar. Esta es una concepción compartida por Avanza Chile, tal como pudimos rastrear en varias columnas que elogiaban los avances en la materia del primer gobierno de Piñera:

Mujer y maternidad son temas importantes para Chile, por ello la administración anterior, aumentó los tratamientos contra la infertilidad, hizo programas de apoyo a madres adolescentes, aumentó las mamografías, principalmente en zonas rurales, y extendió el posnatal a 6 meses. Sin embargo, aún quedan temas pendientes con las mujeres (...) Sala Cuna universal, que hoy le permitiría a más de 500 mil mujeres incorporarse al trabajo, Reforma a la Sociedad Conyugal, que reconoce a la mujer casada capacidad para administrar sus propios bienes. Reforma por Violencia en el Pololeo (parejas sin convivencia). Reforma a las Isapres. (Avanza Chile, 2017).

En sus tiempos como experta de Avanza Chile, la actual ministra de deporte Isabel Plá, fue enfática al criticar las deudas del gobierno de Bachelet con los derechos de las mujeres y en particular, con el logro de su autonomía económica. Según Plá:

La incorporación de la mujer al mundo del trabajo tiene un impacto que va muchísimo más allá del estrictamente económico. Consagrar la igualdad de derechos y oportunidades en la ley, protegerla de los abusos y de la violencia intrafamiliar, crear un Ministerio de la Mujer, llamarlas a consolidar su autoestima, a tener seguridad en sus capacidades y a superar su propia carga machista, resulta insuficiente. La mitad de las chilenas no tiene hoy autonomía económica, que es, en la práctica, la que les permitiría tomar decisiones personales con mayor independencia y libertad para definir sus vidas. (Avanza Chile, 2016). 
El pedido por una mayor autonomía económica para las mujeres es la recomendación más recurrida de los TTD. Lo que varía entre un centro y otro, es el tipo de medida a través de la cual se alcanzaría dicho objetivo. Mientras que los expertos de Avanza Chile señalaron en mayo de 2016 que abogaban por una intervención subsidiaria del Estado, los analistas de LyD anteponen el rol del mercado y solicitan que las políticas públicas velen "por remover los obstáculos para que las mujeres puedan libremente optar a desenvolverse en los distintos sectores de la sociedad" (LyD, 2020c).

\section{Participación sin representación}

Dentro de sus recomendaciones para la gestión estatal, LyD señala que en la sociedad chilena "los aspectos prioritarios en que se requiere de mayor apoyo (...) se dan en relación con facilitar aún más la inserción de la mujer en el mercado laboral y el tema del cuidado infantil" (Camhi, 2019, p. 28). En un nivel de menor importancia se halla la baja participación de la mujer en las esferas de decisión política y empresarial. No obstante lo anterior, encuentra incorrectos "los instrumentos de discriminación positiva usados para lograr una mayor integración de mujeres en estos ámbitos, como las cuotas de género obligatorias ..., ya que muchas veces terminan vulnerando el principio de igualdad universal". Así, para LyD "mujeres más educadas y empoderadas, atendiendo a sus preferencias personales, debieran poder acceder con el tiempo en mayor medida a dichos cargos" (Camhi, 2019, p. 29).

Tras la aprobación de la ley de paridad en el Congreso, LyD manifestó su desacuerdo argumentando que las cuotas de género debilitan la democracia al alterar el resultado del proceso eleccionario: "Lo que se requiere es promover la participación de las mujeres, pero no a costa de torcer la voluntad democrática" (LyD, 2020a). Meses antes, la directora de Políticas Públicas de LyD, manifestó una opinión coincidente, arguyendo que la ley de cuotas atenta contra el principio de igualdad de derechos y contra la dignidad de las mujeres:

Lo que insulta a las mujeres es que no nos dejen competir con igualdad. Como todos los seres humanos, queremos y podemos validarnos por nuestros propios méritos. Hay que advertir el peligro que el abuso del sistema de "cuotas" termine consolidando un "machismo 2.0", una actitud condescendiente que castiga y descali- 
fica a priori a la mujer que se integra a un ambiente laboral "porque había que cumplir la cuota." (Equipo Tendencias, 2018).

También los expertos de FPP han expresado sus reservas hacia las políticas afirmativas. En una interpretación propia que han denominado feminismo liberal dicen anhelar la justicia sin privilegios y la igualdad ante la ley de todos los individuos y asumen que la discriminación positiva genera una falsa sensación de justicia y desconoce la igual dignidad de todos los seres humanos, ya que el Estado establece, arbitrariamente, categorías de ciudadanos (FPP, 2019). El feminismo liberal ha sido la respuesta de este TTD a la ola de movilizaciones en Chile que - en el marco de la guerra de ideas contra el progresismo y la izquierda - contraataca envolviendo al feminismo dentro del modelo neoliberal de libre mercado.

La táctica seguida por la FPP, encabezada por su director Axel Kaiser, ha consistido en la creación de un campo de antagonismo entre un "feminismo liberal y científico, que busca la igualdad jurídica y cultural de la mujer y que ... reconoce los hallazgos científicos que han demostrado que existen varias diferencias de género producto de cómo están cableados nuestros cerebros" y un "feminismo marxista que concibe la relación del hombre y la mujer como una entre enemigos donde una es siempre víctima del otro" (Fernández, 2018). La segunda corriente, es descrita como "intolerante y autoritaria, promotora de la censura, las funas, la violencia en contra del hombre y en contra de la mujer que rechace sus postulados" (Fernández, 2018).

Como fuentes teóricas de este feminismo liberal y científico, Kaiser apela a la autoridad de Mary Woolstonecraft, Camille Paglia, Deirdre Mccloskey, Roxana Kreimer y María Blanco. Esta última, es una economista española que integra el Instituto Juan de Mariana, dedicado a la difusión de las ideas de la Escuela de Salamanca, y que ha sido invitada en varias ocasiones por la FPP para hablar sobre la compatibilidad entre capitalismo y feminismo y el "falso" rumbo adoptado por los "feminismos de izquierda radicalizada".

\section{Libertad sin reconocimiento}

Según el Decálogo del feminismo liberal elaborado por la FPP, sobre la base de la obra de Blanco y frases sueltas de las referentes menciona- 
das, la corriente liberal y científica cree en el entendimiento y la complementariedad de hombres y mujeres y es contrario a la idea de la lucha de géneros (FPP, 2019), con lo cual suspende toda la conflictividad derivada del sistema de jerarquías del orden heteropatriarcal.

En el documento se sostiene, además, que el feminismo liberal fomenta una cultura de autonomía y responsabilidad por sobre una cultura de la victimización y agrega que la victimización, como cultura de atribuir todas las responsabilidades a otros, es el mayor obstáculo para superar problemas. "Victimizarse es la mejor manera de encadenarse y no avanzar" (FPP, 2019, líneas 20-21). La estratagema del TT, consiste en asociar las demandas de justicia de las mujeres con la autovictimización e incluso con la mentira.

La negación de una situación estructural de injusticia es recurrente en las intervenciones de FPP y no es exclusiva de las temáticas de mujer y género. En su momento, otro experto de este think tank calificó la creación del Ministerio de la Mujer y la Equidad de Género, como "una falsa o aparente protección de una especie en el género de la tolerancia y el respeto a la diversidad y que explica la poca utilidad que un ministerio como el planteado realmente representa para el país" (Infante, 2015, párrafo 1). En sintonía con la doxa del Estado mínimo defendida por FPP, para este analista cualquier iniciativa estatal en favor de los derechos de las mujeres constituye un avance pernicioso sobre la libertad de los individuos y una expansión burocrática inaceptable:

¿Qué ha logrado el Sernam ${ }^{22}$ ? Además de algunas medidas absurdas -como el instalar el aberrante concepto de "femicidio"-, poco se ha avanzado. La incorporación de la mujer a la fuerza laboral es un hecho que se logró no gracias a políticas públicas, sino finalmente por la necesidad del mercado de contar con más trabajadores —sí, el perverso mercado-, y la paridad de sueldos se logrará precisamente por las mismas necesidades (por si a alguien aún no le cae el tejo, ante la escasez de trabajadores, suben los sueldos). (Infante, 2015, párrafo 7).

El octavo punto del Decálogo del feminismo liberal converge con los enunciados de Infante en la perspectiva de instalar la idea de un femi-

22 Servicio Nacional de la Mujer. 
nismo compatible con el neoliberalismo: "El libre mercado ha sido el sistema que más ha favorecido la liberación de las fuerzas productivas y creativas de las mujeres y de la sociedad en general, para que puedan progresar y desarrollarse en la medida que quieran" (FPP, 2019, líneas 61-62).

En tanto arma discursiva, el feminismo liberal ha extendido su radio de acción más allá de los asuntos de las mujeres para responder a los colectivos que demandan un desmonte del esquema de injusticias en Chile. El punto 6 del decálogo reza: "El feminismo liberal cree que la identidad y derecho son individuales, no colectivos". Y a continuación, ofrece un desarrollo que no es una explicación lógica del enunciado: "La identidad de una persona no se reduce a una sola categoría de análisis como lo es el género, la clase, la condición sexual u orientación política" (FFP, 2019, líneas 46-47). Estas dos premisas rematan con el siguiente corolario "Hay visiones del feminismo que reducen a las mujeres solo a su género dejando fuera las múltiples dimensiones que tiene cada ser humano" (FPP, 2019, líneas 47-48).

Este rechazo de los derechos colectivos emerge en momentos en los que el feminismo plurinacional chileno incorpora dentro de sus vindicaciones los derechos de los pueblos originarios, el antirracismo, la defensa del territorio, la soberanía alimentaria, los derechos de los migrantes, las disidencias sexuales y la educación no sexista. La argucia de proponer que la identidad de una persona no se reduce a su condición de género, clase, condición sexual u orientación política, avanza en el objetivo de restar legitimidad a estas luchas colectivas negándoles cualquier reconocimiento.

Yendo a los pronunciamientos en materia de derechos sexuales y (no) reproductivos observamos que los tres TTD coinciden en una postura contraria al aborto, en línea con los congresistas de la coalición de Chile Vamos. Argumentos como la primacía de los derechos del "niño por nacer", el derecho a la objeción de conciencia institucional en oposición a esta práctica, se desplegaron en media decena de columnas publicadas el mismo año en que se aprobó la ley de despenalización en tres causales.

Para los libertarianos de la FPP, "no existe una sola posición liberal frente al tema (...) en el liberalismo originario o clásico se puede 
ser partidario o contrario al aborto" (Blanco, 2017, párrafo 1). De este modo,

se puede ser liberal y contrario al aborto porque lo decisivo es el respeto por derechos de terceros. Si existe la convicción de que hay derechos desde la concepción que no pueden ser afectados, entonces se puede ser contrario al aborto y liberal. (Blanco, 2017, párrafo 7).

Tras la aprobación de la ley de aborto legal, LyD celebró una sentencia de la Corte chilena que permitió la objeción de conciencia institucional a las instituciones médicas privadas que no desearan cumplir con la ley de interrupción legal del embarazo. Según una investigadora del programa legislativo de este TT, la sentencia "resguarda la autonomía de los cuerpos intermedios, fundamental para una sociedad libre, en la que las personas se puedan agrupar e imprimir sus convicciones a sus asociaciones y no ser coaccionadas por el Estado a través de ellas" (LyD, 2019).

El piñerismo nucleado en Avanza Chile fue más directo en su postura y se movió de su pretendido centro para suscribir una postura abiertamente conservadora. Al momento de la presentación del proyecto por el gobierno Bachelet, Piñera fue enfático en la idea de que sobre el derecho al aborto debe primar el derecho a la vida del niño. Además de razones morales, jurídicas y prácticas, Piñera esgrimió una cuarta razón de naturaleza religiosa:

Dada la tradición y mayoría de cristianos en la sociedad chilena, es importante considerar la concepción de los cristianos sobre el origen de la vida. Para los cristianos los seres humanos somos creados a imagen y semejanza de Dios. La vida es un don de Dios. Solo Él tiene el poder de darla y el derecho a quitarla. Por eso, debemos proteger la vida y dignidad humana desde su concepción hasta su muerte natural. Y, por lo mismo, oponernos a la eutanasia y a la pena de muerte. (Avanza Chile, 2014, párrafo 7).

Los textos en torno a las temáticas de mujer y género producidos por Avanza Chile persiguen un doble objetivo: por un lado, contemporizar con algunas demandas de las mujeres chilenas tales como la lucha contra la violencia de género y la incorporación laboral y, por otro, hacer oposición al gobierno de Bachelet marcando su deuda con 
las mujeres. Ambas tácticas hicieron parte de la estrategia piñerista de encontrar un nuevo centro social.

Isabel Plá, otra integrante de Avanza Chile, hizo eco de la postura antiaborto de Piñera en una crítica a la gestión del Sernam de Bachelet. Según Plá: "Su agenda es pobre, sobreideologizada, inspirada en consignas que ya suponía superadas y está concentrada, básicamente, en la despenalización del aborto, al que convirtió artificialmente en la conquista más simbólica de las chilenas" (Avanza Chile, 2016, párrafo 6).

Plá se incorporó al gabinete de Piñera en 2018. Su paso por el Ministerio de la Mujer y la Equidad de Género fue corto, polémico y muy criticado por el movimiento feminista plurinacional chileno, al que confrontó desde el inicio. Así, dijo en relación a la Huelga Nacional Feminista del $8 \mathrm{M}$ que era "una convocatoria de un sector de la oposición con una agenda que excede los temas de la mujer" (CNN Chile, 2019). Sin embargo, al ver el impacto de las movilizaciones, Plá cambió su parecer y celebró la marcha. Otro evento que impactó en la opinión pública fue el doble rasero de la ministra en relación con la violencia sufrida por las mujeres chilenas durante los estallidos sociales de 2019: mientras envió un saludo a las carabineras quemadas por una bomba molotov en Plaza Italia, se llamó al silencio con los cientos de mujeres que denunciaron agresiones físicas y sexuales de parte de la policía (Román, 2020).

En mayo de 2020, Piñera nombró como ministra de la Mujer y la Equidad de género a Macarena Santelices, una militante y exdirigente de la ultraderechista Unión Demócrata Independiente (UDI), sobrina nieta del dictador Augusto Pinochet, y sin ninguna formación o experiencia en materia de políticas de género. En el acto de nombramiento, el presidente Piñera emitió un discurso con el que buscó suspender la conflictividad inherente al orden de género, ignorándola:

Esta no es una lucha o un conflicto entre hombres y mujeres, esta es una lucha de hombres y mujeres, juntos, para construir una sociedad en la que tengamos plena y total igualdad de derechos, deberes y oportunidades (...) Estoy seguro que Macarena va a estar a la altura de este tremendo desafío. (Ámbito, 2019, párrafos 5 y 6). 


\section{Conclusiones}

A lo largo de este trabajo quisimos aportar al conocimiento de los think tanks de derecha en Chile. En el primer apartado, avanzamos en una caracterización de la trayectoria y perfil ideológico de tres de ellos: Libertad y Desarrollo, Fundación para el Progreso y Avanza Chile. De ideología liberal-conservadora, LyD es el más antiguo de los tres, con un perfil orientado a la asesoría en políticas públicas según demanda, por lo que podemos clasificarlo, siguiendo a Weaver (1989), como un instituto de investigación bajo contrato. Por su parte, FPP es un centro de estudios libertariano de reciente creación que enfoca su trabajo en la formación de jóvenes bajo este sistema de ideas pero, sobre todo, en la guerra cultural contra el progresismo y la izquierda, doble condición que nos inclina a catalogarlo como un centro militante. Finalmente, Avanza Chile, de tendencia liberal centrista, es un TT cuya creación obedece a la necesidad de realineamiento de los expertos y cuadros de la coalición de derecha que acompañó a Sebastián Piñera en las elecciones de 2018, por lo que se trata de un TT organizado en torno a un partido político: Chile Vamos.

En el segundo apartado, analizamos los discursos de estos TTD en torno a las temáticas de mujer y género. Ordenamos su repertorio alrededor de tres demandas transversales de los feminismos contemporáneos: la redistribución económica, la representación política y el reconocimiento de derechos. Tras el análisis de informes, columnas de opinión y apariciones en medios de comunicación de los integrantes de estas usinas, encontramos que los combates de la derecha chilena por reposicionarse en el mercado de ideas han hecho de estos TT lugares de producción y resonancia de enunciados que buscan dar la apariencia de cientificidad y juicio razonado pero que son, en su mayoría, artefactos publicitarios que buscan un impacto rápido y altamente persuasivo entre la opinión pública.

Comúnmente abocados a temáticas vinculadas con la economía, el derecho y la seguridad, estos TT empezaron a producir discursos en torno a los derechos de las mujeres, en reacción a la ola feminista que ha atravesado al país en la última década y a los avances en la materia logrados en la segunda administración de Michelle Bachelet. Cabe destacar que las demandas de las disidencias sexo-genéricas raramente son tematizadas por estos TT. En consonancia, la "Agenda Mujer" 
del presidente Piñera, ofrece intervenciones estatales dirigidas exclusivamente a las féminas, desestimando las demandas y el peso simbólico adquirido por los colectivos LGTBIQ en el país.

La estrategia adoptada para librar la guerra de ideas contra el progresismo y la izquierda ha consistido en adoptar, de manera parcial y selectiva, las banderas del feminismo buscando hacerlas compatibles con la doxa neoliberal. En relación con las demandas de redistribución económica, la batalla se trasluce en la preocupación por promover políticas de acceso de las mujeres al mundo laboral y atender parcialmente a la problemática del cuidado infantil, sin discutir la división sexual del trabajo, los mecanismos estructurales de discriminación y segregación que operan en el mercado laboral, ni las condiciones sistémicas de un modelo neoliberal que limita, de suyo, las oportunidades laborales de las mujeres. Por otra parte, los diagnósticos y medidas recomendados en materia de empleo, las jerarquizan como trabajadoras secundarias y refuerzan los estereotipos de género.

En cuanto a la representación política, en los discursos analizados no hay mención a mecanismos concretos para promover la participación equilibrada entre mujeres y hombres. En su lugar, las intervenciones tratan de suspender la desigualdad consustancial al orden de género ignorándola e instalando en su lugar enunciados de corte "consensual" como el "falso enfrentamiento entre hombres y mujeres" promovido por cierto feminismo; el carácter complementario de hombres y mujeres que trabajan "codo a codo", etc. Así, niegan legitimidad a cualquier política afirmativa a través de ideas como que "las mujeres solo se empoderan en ausencia de obstáculos impuestos por el Estado", o que las cuotas en los cargos públicos les restan dignidad y atentan contra el derecho a la igualdad.

No obstante la carencia de políticas de redistribución económica, representación política y reconocimiento simbólico en favor de las mujeres, estos TT insisten en la compatibilidad entre feminismo y neoliberalismo. Pero esta reconciliación encuentra sus límites cada vez que sus expertos hacen recomendaciones de política pública que mantienen adscriptos los derechos de las mujeres a sus funciones de esposas y madres. Un feminismo que suscribe la desigualdad como un dato natural, que parte de un modelo de familia nuclear y bipa- 
rental, dejando incólume la división sexual del trabajo, que interpela exclusivamente a las mujeres cis blancas que deciden maternar y deja afuera otros proyectos de vida, otros arreglos familiares y otros sujetos de derechos como los pueblos originarios y las disidencias sexo-genéricas. En fin, un feminismo que pacta con el orden de desigualdad y discriminación existente, solo puede llamarse feminismo al precio de su adjetivación. Demos la bienvenida al "feminismo colaboracionista".

\section{Referencias}

Alenda, S., Foulon, C. Le, y Suárez-Cao, J. (2019). La batalla por las ideas en tiempos posideológicos: Adaptaciones y permanencias ideológicas en la nueva centroderecha chilena. Revista de Sociologia e Política, 27(70), e004. https://doi. org/10.1590/1678-987319277004

Althusser, L. (1988). “Ideología y aparatos ideológicos del Estado”. En L. Althusser (Ed.), La filosofía como arma de la revolución (pp. 97-141). México, D.F.: Ediciones Pasado y Presente.

Álvarez, S. L. y Navarrete, A. B.. (2019). Cronología del movimiento feminista en Chile 2006-2016. Revista Estudos Feministas, Florianópolis, 27(3), e54709. Recuperado de https://www.scielo. br/scielo.php?pid=S0104-026X2019000300216\&script=sci_ arttext\&tlng=es

Ámbito (6 de mayo de 2020). Piñera nombra ministra de la Mujer a una sobrina-nieta de Pinochet y desata la polémica. Recuperado de https://www.ambito.com/mundo/chile/pinera-nombraministra-la-mujer-una-sobrina-nieta-pinochet-y-desata-lapolemica-n5100666

Avanza Chile (2020). ¿Quiénes somos? Recuperado de http://www. avanzachile.cl/quienes-somos/

Avanza Chile (4 de mayo de 2016). \#ChilePuedeMás: Radiografía del empleo y oportunidades para las mujeres. [audiovisual]. Recuperado de https://www.avanzachile.cl/avanza-tv/chilepuedemas-radiografia-del-empleo-y-oportunidades-paralas-mujeres/

Avanza Chile (8 de marzo de 2017). Las deudas del Gobierno con las mujeres. Recuperado de http://www.avanzachile.cl/noticia/ las-deudas-del-gobierno-con-las-mujeres/ 
Avanza Chile (14 de junio de 2014). Piñera, Sebastián: "Derecho a nacer vs Derecho a abortar". Recuperado de http://www.avanzachile.cl/columna/derecho-nacer-vs-derecho-abortar/

Avanza Chile (18 de marzo de 2016). Isabel Plá. ¡Trabajo para las mujeres! Recuperado de https://www.avanzachile.cl/columna/ trabajo-para-las-mujeres/

Blanco, B. (13 de junio de 2017). Axel Kaiser, principal exponente del liberalismo en Chile: "Se puede ser liberal y contrario al aborto". Emol.com. Recuperado de https://www.emol.com/ noticias/Nacional/2017/06/13/862409/Axel-Kaiser-principal-exponente-del-liberalismo-en-Chile-Se-puede-ser-liberal-y-contrario-al-aborto.html

Camhi, R. (2019). Mujer y desarrollo en Chile. Serie Informe Social $N^{\circ}$ 178 julio, 2019. Recuperado de https://lyd.org/wp-content/ uploads/2019/08/serie-informe-social-178-julio.pdf

CNN Chile (28 de febrero de 2019). Ministra de la Mujer se desmarca del llamado a huelga para el $8 \mathrm{M}$ y asegura que parece una convocatoria "de la oposición". Recuperado de: https://www. cnnchile.com/pais/ministra-mujer-desmarca-huelga-8moposicion_20190228/

Cociña, M. y Toro, S. (2009). Los think tanks y su rol en la arena política chilena. En E. Mendizábal y K. Sample (Eds.), Dime a quién escuchas... Think tanks y partidos políticos en América Latina (pp. 98-126). Estocolmo: IDEA Internacional \& ODI.

Domínguez, J. I. (ed.). (1996). Technopols: Freeing Politics and Markets in Latin America in the 1990s. University Park, PA: Penn State University Press.

Encuentro Plurinacional de Mujeres que luchan (2019). Programa, carácter y objetivos de la Huelga general feminista 8 de marzo de 2019. Recuperado de https://issuu.com/cf8m/docs/sintesis_encuentro_plurinacional_de/8

Equipo Tendencias (25 de mayo de 2018). Bettina Horst: Cuidado con el "machismo 2.0". La Tercera. Recuperado de https://www. latercera.com/tendencias/noticia/bettina-horst-cuidadomachismo-2-0/179103/

Fernández, M. E. (22 de mayo de 2018). El feminismo según Axel Kaiser: "Jamás en la historia la situación de la mujer ha sido mejor que hoy". La Tercera. Recuperado de https://www.latercera.com/la-tercera-pm/noticia/feminismo-segun-axel- 
kaiser-jamas-la-historia-la-situacion-la-mujer-ha-mejorhoy/174605/

Fischer, K y Plehwe, D. (2013). La formación de la sociedad civil neoliberal en América Latina: redes de think tanks e intelectuales de la nueva derecha. Nueva Sociedad, 145. Recuperado de https://nuso.org/articulo/redes-de-think-tanks-e-intelectuales-de-derecha-en-america-latina/

Fraser, N. (2008). Escalas de justicia. Barcelona: Herder.

Fundación para el Progreso, FPP. (2019). Decálogo del feminismo liberal. Recuperado de https://fppchile.org/es/blog/decalogodel-feminismo-liberal/

Fundación para el Progreso, FPP. (2020). Nuestra misión y nuestro propósito. Recuperado de https://fppchile.org/es/misionvision-y-valores/

Gárate, M. (2008). Think tanks y centros de estudio. Los nuevos mecanismos de influencia política en el Chile post-autoritario. En M. de Cea P., Díaz, y G. Kerneur (Eds.), Chile: ¿De país modelado a país modelo? Una mirada sobre la política, lo social y la economía (pp. 67-85). Santiago de Chile: LOM Ediciones.

Gartenlaub G. A. (2018). Trayectorias, permanencias y continuidades de la competencia política y de los perfiles ideológicos de los partidos de derecha chilenos en la posdictadura (Tesis doctoral). Universidad de Chile, Santiago, Chile. Recuperado de http://repositorio.uchile.cl/handle/2250/173237

González Bustamante, B. (2013). El estudio de las élites en Chile: aproximaciones conceptuales y metodológicas. Intersticios sociales, 6, 1-20. Recuperado de http://www.scielo.org.mx/scielo. php?script=sci_arttext\&pid=S2007-49642013000200004\&lng =es\&tlng=es.

Infante, J. (2015). Sobre instituciones de más y la utilización de la mujer. Recuperado de https://fppchile.org/es/sobre-institucionesde-mas-y-la-utilizacion-de-la-mujer/

Jerez, C. (18 de diciembre de 2018). ¿Quiénes son los Von Appen?: La familia de origen nazi y dueña de Ultramar que tiene en jaque a los trabajadores portuarios. El Desconcierto. Recuperado de https://www.eldesconcierto.cl/2018/12/18/quienes-sonlos-von-appen-la-familia-de-origen-nazi-y-duena-de-ultramar-que-tiene-en-jaque-a-los-trabajadores-portuarios/ 
Joignant, A. y Güell, P. (2011). Notables, tecnócratas y mandarines. Elementos de sociología de las élites en Chile (1990-2010). Santiago de Chile: Ediciones Universidad Diego Portales.

Kaiser, A. (2011). La madre de todas las batallas. Recuperado de https://www.libertaddigital.com/opinion/ideas/la-madrede-todas-las-batallas-1276239453.html

Libertad y Desarrollo, LyD. (2016). ¿Cuánto ha aumentado la tasa de ocupación de las mujeres con el programa Bono al Trabajo a la Mujer? Serie Informe Social, 161, Recuperado de https:// lyd.org/wp-content/uploads/2017/01/SISO-161-Cuantoha-aumentado-la-tasa-de-ocupacion-de-las-mujeres-conel-programa-Bono-al-Trabajo-de-la-mujer-Octubre2016. pdf

Libertad y Desarrollo, LyD. (2018). Ministerio de Familia y desarrollo social: Redefiniendo el foco de la política social. Temas Públicos, 1364, 2. Recuperado de https://lyd.org/wp-content/ uploads/2018/09/TP-1364-MINISTERIO-DE-FAMILIA-YDESARROLLO-SOCIAL.pdf

Libertad y Desarrollo, LyD. (2019). Objeción de conciencia institucional: reconocimiento a la autonomía de los cuerpos intermedios. Recuperado de https://lyd.org/centro-de-prensa/ noticias/2019/02/objecion-de-conciencia-institucional-reconocimiento-a-la-autonomia-de-los-cuerpos-intermedios/

Libertad y Desarrollo, LyD. (2020a). Paridad de género: en desmedro de la democracia. Temas Públicos, 1437(1). Recuperado de https://lyd.org/wp-content/uploads/2020/03/tp-1437-paridad-de-genero.pdf

Libertad y Desarrollo, LyD. (2020b). Orientaciones a investigadores de LYD. Recuperado de https://lyd.org/wp-content/ uploads/2014/10/Orientaciones-a-Investigadores-LYDWeb-nuevo-logo.pdf.

Libertad y Desarrollo, LyD. (2020c). Día Internacional de la mujer: ¿cómo estamos? Recuperado de https://lyd.org/centro-deprensa/destacados/2020/03/dia-internacional-de-la-mujer-como-estamos/

Mira, A. (2011). Crisis de representatividad y estallido social: una aproximación a la actual experiencia chilena. Polis, 10(30), 185197. https://dx.doi.org/10.4067/S0718-65682011000300009 
Moreno, M. (2015). Think tanks en Chile: estilos y prácticas tecnocráticas en la formación de políticas. Revista Enfoques, 8(12), 103-125. Recuperado de https://revistaenfoques.cl/index. $\mathrm{php} /$ revista-uno/article/view/155

Olivares, L., A., González-Bustamante, B., Meneses, J., y Rodríguez, M. (2015). Los think tanks en el gabinete: una exploración del caso chileno (2006-2014). Revista de Sociología, 29. Recuperado de https://revistadesociologia.uchile.cl/index.php/ RDS/article/view/36177

Pinilla, P. J. (2012). Think tanks, saber experto y formación de agenda política en el Chile actual. Polis, Santiago, 11(32), 119-140. https://dx.doi.org/10.4067/S0718-65682012000200008

Reyes, C. (9 de agosto de 2018). Ojalá tengamos más Estados y más bingo: Varela responde a Bachelet y desata polémica. La Tercera. Recuperado de https://www.latercera.com/nacional/ noticia/ojala-tengamos-mas-estados-mas-bingos-varelaresponde-bachelet-desata-polemica/275707/

Rodríguez, G.P. (2013). Hacia un modelo integral de ciudadanía. El debate liberal-comunitarista y los retos del republicanismo. Utopía y Praxis Latinoamericana, 18(63), 75-89. Recuperado de https://www.redalyc.org/pdf/279/27937086007.pdf

Román, C. (2020). Más de la mitad sin cumplir: los pendientes de la Agenda Mujer. Recuperado de https://www.pauta.cl/politi$\mathrm{ca} /$ mas-de-la-mitad-sin-cumplir-agenda-mujer-de-cara-al8-de-marzo-2020

Rovira Kaltwasser, C. (2019). La (sobre)adaptación programática de la derecha chilena y la irrupción de la derecha populista radical. Colombia Internacional, 99, 29-61. https://dx.doi. org/10.7440/colombiaint99.2019.02

Schuster, S. y Morales, F. (2015). La dimensión ideológica de las políticas públicas en el gobierno de Sebastián Piñera. Revista Uruguaya de Ciencia Política, 24(1), 49-70. http://www. scielo.edu.uy/scielo.php?script=sci_arttext\&pid=S1688499X2015000100005

Silva, P. (2010). En el nombre de la razón: tecnócratas y política en Chile. Santiago de Chile: Ediciones Universidad Diego Portales.

Torres, C. (Coord.) (2011). Miradas y reflexiones feministas: Sebastián Piñera, año uno: conmociones y exigencias sociales. Santiago 
de Chile: Fundación Instituto de la Mujer y Fundación Heinrich Böll Cono Sur.

Varas, A. (2013). El Gobierno de Piñera (2010-2014). La fonda aristocrática rediviva. Santiago de Chile: Catalonia.

Weaver, K. (1989). The changing world of think tanks. Political Science and Politics, 22(3), 563-578. https://doi.org/10.1017/ s104909650003105x 